Decay Problems in Mathematical Physics.

CoNsIDER a quantity $u$, satisfying the equation :

$$
L(u)=\rho \frac{\hat{c}^{2} u}{\partial t^{2}}+w \frac{\partial u}{\partial t},
$$

in a closed finite space, with any homogeneous boundary condition at the boundary of the aforesaid space.

Here $L$ is a linear differential operator of the second order, extending to any number of dimensions; for example, for three dimensions :

$$
L(u) \equiv \frac{\partial}{\partial x}\left(p \frac{u}{\partial x}\right)+\frac{\partial}{\partial y}\left(p \frac{\hat{c} u}{\partial y}\right)+\frac{\partial}{\partial t}\left(p \frac{\partial u}{\partial t}\right)-q u,
$$

whereas $\rho$ and $w$ are finite and positive functions of the independent variables entering in $L$

Then the following theorem has been proved: If a system at rest, the free motion of which is described by (1), is acted on by an oscillatory force of frequency large with respect to the lowest natural frequency of the system, until the stationary state is reached, the time of increase is directly proportional to the whole absorption and indirectly proportional to the volume, but independent of the shape of the system and of the places of the experimenter and the force. If the force, after the stationary state is reached, stops abruptly, the time of decay obeys the same law. Moreover, the increase is complementary to the decay, the sum of the amplitudes at a time $t$, measured from the moment the force starts in one case and from the moment the force stops in the other, being constant and independent of $t$.

This theorem may immediately be applied to the theory of electromagnetic radiation in closed spaces and to the acoustics of large rooms. In both cases it gives the time required by the system to reach the stationary state after a change of the radiating forces.

It has been verified experimentally by W. Sabine in the acoustics of large rooms ("Collected Papers on Acoustics", p. 39), whilst experiments by Dr. Zwikker in this laboratory have shown that the complementary nature of increase and decay really exists.

An extension, comprising absorption $w$, which depends on the frequency, but in a bounded and for high frequencies slowly variable way, may easily be proved.

For details reference is made to a paper, entitled : " Über das Dämpfungsproblem der mathematischen Physik", which will be published in the Mathematische Annalen and to a paper entitled "On the Acoustics of Large Rooms", which will appear shortly in the Phil. Mag.

Natuurkundig Laboratorium der

N. V. Philips' Gloeilampenfabrieken, Eindhoven.

\section{Raman Effect in Gases and Liquids.}

IN a note recently communicated to NATURE it was pointed out that the Raman effect in triatomic molecules having a linear model could be explained on the assumption of a fundamental mode of vibration along the line joining the three nuclei of the molecules. Such vibrations take place along the direction of propagation of the radiation and behave as optically inactive ones and are not manifested in the near infra-red bands. The case of carbon dioxide was discussed on this basis.

The Raman effect of liquid carbon disulphide has recently been studied in Sir C. V. Raman's laboratory, and it shows two prominent modified lines due to absorption of energy corresponding to infra-red wave lengths $12.5 \mu$ and $15.27 \mu$ (private communication), which have so far not been observed as absorption bands. This can now be explained on the supposition of an optically inactive frequency $\nu=728 \mathrm{~cm}^{-1}$ of $\lambda=13.73 \mu$. The two lines being due to transitions $\Delta i= \pm 1$ of the molecule (Nat. Research Council Bull., 57, p. 62) from this fundamental vibrational state.

The observations of McLennan and McLeod (NATURE, Feb. 2, 1929) regarding the Raman effect in liquid oxygen, nitrogen, and hydrogen, showing the absor'ption of energy corresponding to values of $\nu=1554 \mathrm{~cm}^{-1}$, $\nu=2335 \mathrm{~cm} .^{-1}$, and $\nu=4149 \mathrm{~cm}^{-1}$, also suggest that the nuclei of these homopolar molecules even in the liquid state execute vibrations under impressed radiation, though no bands in these regions have so far been observed. One may thus be led to imagine that nuclear vibrations occur along the direction of propagation of the radiation and are thus optically inactive so far as absorption is concerned. Dennison (Astro. Jour., 62, 1925) calculated the fundamental frequencies of the methane molecule from dynamic considerations, and out of the four fundamental frequencies, three have been identified in the absorp. tion bands and it has been suggested that the fourth one at $\lambda=6.58 \mu$ is optically inactive. The presence of such an optically inactive vibration is also suggested from the work of Bennet and Meyer on the spectra of methyl halides (Phys. Rev., 33, 895; 1929). It will be worth while to study the Raman effect of this gas to ascertain from the modified lines the presence of this fundamental frequency suggested from dynamical considerations.
P. N. Ghosh.

P. C. Mahanti.
Applied Physics Laboratory, University College of Science, Calcutta, May 27.

The Raman Effect for X-rays.

IN a letter in NATuRE of April 27, p. 642, we reported some experiments on the fine structure of the normal scattered molybdenum $K a$-radiation. From our photometer curves it is clearly shown that the normal scattered molybdenum radiation from graphite has exactly the same structure as the direct radiation from a molybdenum target or, in other words, that no Raman effect exists. An analogous result was obtained by W. Ehrenberg (Zeit. f. Physik, $53,234 ; 1929)$; whereas B. Davis and D. P. Mitchell (Phys. Rev., 32, 331 ; 1928) obtained on the contrary a much more complicated structure in the case of the normal scattered radiation.

In the meantime the experiments were continued by D. P. Mitchell (Phys. Rev., 33, 871; 1929), and analogous results to those obtained with graphite are reported when beryllium and aluminium were used as scattering substances. Though for the moment we cannot explain the difference between Mitchell's results and our own, we should like to direct attention to the following fact: In the case of beryllium Mitchell finds an 'anti-Stokes line' which should be scattered by an atom in the excited $L$-state. Now a simple calculation shows that even when working with an energy so large as $3 \mathrm{kw}$., and such short distances as was done by Mitchell, much less than one beryllium atom in $10^{15}$ can be in the excited $L$-state. Hence it follows that it is quite impossible to account for the order of magnitude of the intensity of the anti-Stokes line quoted by Mitchell.

\section{Coster.}

I. NitTA.

W. J. ThiJssen

Natuurkundig Laboratorium der

Rijks-Universiteit, Groningen. 